Tautomeria de Valência em Complexos de Cobalto

\author{
de Morais, A. C. P.*
}

Rev. Virtual Quim., 2015, 7 (5), 1663-1676. Data de publicação na Web: 6 de setembro de 2015

http://www.uff.br/rvq

\title{
Valence Tautomerism in Cobalt Complexes
}

\begin{abstract}
Valence tautomeric (VT) complexes have attracted considerable attention due to their potential application in molecular electronic devices. This class of complexes exhibits thermally and/or photoinduced rearrangements associated with an intermolecular electron transfer between the redox active ligand and the metal ion forming two isomers of similar energy. The nature of the electroactive ligand and the type of external stimulus (light, temperature, or pressure) are critical to the generation of the two electronic isomers. Although several VT complexes have been described in the literature, the search continues for species with required properties for practical applications. The aim of this article is to present some of the most relevant complexes thus far published, while discussing the main properties that a compound should present in order to become a candidate for the development of molecular devices.
\end{abstract}

Keywords: Valence tautomerism; molecular electronic devices; coordination compounds.

\section{Resumo}

Complexos com tautomeria de valência (TV) têm atraído muita atenção recentemente por serem potenciais candidatos para aplicação no desenvolvimento de dispositivos eletrônicos moleculares. Esta classe de complexos exibe rearranjos induzidos termicamente e/ou fotoinduzidos, associados a uma transferência eletrônica intramolecular reversível entre o ligante redox coordenado e o íon metálico, formando dois isômeros eletrônicos. A natureza do ligante eletroativo e o tipo de perturbação externa (luz, temperatura, ou pressão) são fatores críticos para a geração desses dois isômeros eletrônicos. Embora vários complexos com TV já terem sido descritos na literatura, a busca por espécies que contenham as propriedades necessárias para aplicações práticas continua. Portanto, o objetivo deste trabalho é descrever alguns dos complexos com TV mais relevantes na literatura e abordar as principais propriedades que um composto deve apresentar para que possa ser aplicado no desenvolvimento de um dispositivo molecular.

Palavras-chave: Tautomeria de valência; dispositivos eletrônicos moleculares; compostos de coordenação.

* Universidade Federal Fluminense, Instituto de Química, Departamento de Química Inorgânica, Campus do Valonguinho, CEP 24020-150, Niterói-RJ, Brasil.

$M$ carolprecioso@gmail.com

DOI: 10.5935/1984-6835.20150094 


\section{Tautomeria de Valência em Complexos de Cobalto}

\section{Ana Carolina P. de Morais*}

Universidade Federal Fluminense, Instituto de Química, Departamento de Química Inorgânica, Campus do Valonguinho, CEP 24020-150, Niterói-RJ, Brasil.

* carolprecioso@gmail.com

Recebido em 19 de novembro de 2014. Aceito para publicação em 1 de setembro de 2015

\section{Introdução}

1.1. Microeletrônica e miniaturização

1.2. Materiais eletronicamente lábeis e biestabilidade

2. Tautomeria de valência em complexos de cobalto

3. Estímulos externos que induzem a tautomeria de valência

4. Outros metais na tautomeria de valência

\section{Considerações finais}

\section{Introdução}

\subsection{Microeletrônica e miniaturização}

A microeletrônica é um ramo da eletrônica, voltado para a integração de circuitos eletrônicos, promovendo a miniaturização dos componentes em escala microscópica. Na década de 1950, três americanos inventaram o transistor e a partir desta ideia foi possível a criação do primeiro disco rígido com 5 megabytes de capacidade. $^{1}$ Os primeiros microprocessadores surgiram no início de 1970 e eram constituídos por cerca de 2000 transistores, cujo objetivo era incorporar as funções de uma unidade central de computador em um único circuito integrado. ${ }^{2}$

Ainda em 1965, Gordon Moore, o então presidente da Intel, profetizou com razoável precisão, o ritmo da evolução tecnológica nas quatro décadas seguintes. Sua profecia indicava que o número de transistores nos circuitos integrados dobraria a cada ano. Esse avanço permitiria a criação de máquinas cada vez mais potentes e baratas. Em 1975, Moore revisou sua previsão, ampliando o período para cada dois anos. A estimativa transformou-se em um estatuto da informática e acabou ganhando o nome de lei de Moore, que contribui como parâmetro para uma elevada gama de dispositivos digitais. ${ }^{3,4}$

Em 2003, a Intel previu que a Lei de Moore poderia entrar em colapso entre 2013 e 2018, quando os dispositivos semicondutores de silício avançassem na escala nanométrica em razão das dificuldades geradas pelos problemas de origem térmica e de interferência quântica. ${ }^{5}$ Porém, nos últimos 30 anos, a previsão tem sido estendida e comenta-se que em 2020 os 
dispositivos estarão na escala molecular.

A ideia de se usar moléculas para realizar funções eletrônicas foi apresentada por Aviram e Ratner em 1974. Eles mostraram que uma molécula contendo um grupo doador $\pi$ e outro receptor $\pi$, separados por um espaçador, no caso uma ligação $\sigma$, comporta-se como uma junção $p$-n de um diodo semicondutor. A Figura 1 mostra a molécula utilizada como protótipo, em que as carbonilas retiram densidade eletrônica do anel da quinona e o grupo metóxi aumenta a densidade $\pi$ no anel aromático, tornando-o um sistema aceptor-doador, por meio do qual seria possível a passagem de corrente. ${ }^{6}$<smiles>COc1cc(OC)c(OC)cc1CCC1=CC(=O)C=CC1=O</smiles>

Figura 1. Molécula utilizada por Aviram e Rather como protótipo

Moléculas que podem ser utilizadas como dispositivos em um circuito eletrônico estão sendo estudadas, e algumas delas são baseadas em radicais orgânicos ou complexos mono e multimetálicos eletronicamente lábeis. ${ }^{7}$

\subsection{Materiais eletronicamente lábeis e biestabilidade}

O termo eletronicamente lábil é usado para moléculas e materiais cujas estruturas eletrônicas variam em função de alguma perturbação externa, como temperatura, luz ou pressão. ${ }^{8}$ Tais moléculas são inerentemente biestáveis, ou seja, possuem dois estados não degenerados com diferentes propriedades magnéticas, elétricas e/ou óticas, e por isso podem potencialmente estocar informações em um nível molecular, sendo necessária a ocorrência de histerese para que apresentem memória permanente (não volátil)., ${ }^{9,10}$

Histerese pode ser observada em moléculas que possam existir em duas formas distintas, como, por exemplo, $X$ e $Y$, e essa propriedade é crucial, pois fornece o efeito de memória nos dispositivos. Quando, por exemplo, um campo magnético ou calor é aplicado à espécie $X$, a molécula se torna magnetizada. Para desmagnetizá-la é necessário calor ou campo magnético na direção oposta, gerando, assim, o estado $Y .{ }^{11}$ A histerese ocorre porque as interações intermoleculares da espécie $X$ são diferentes da espécie $Y$ e a transição possui caráter cooperativo. Mas se as interações entre as espécies $X$ e $Y$ forem fracas, a transição é denominada não-cooperativa e a histerese não é observada. ${ }^{10} \mathrm{Um}$ exemplo genérico de histerese pode ser observado na Figura 2, onde o efeito de memória é observado na região entre as curvas, ou seja, nessa região o sistema tem a capacidade de resgatar qual estímulo levou a transição de $X$ para $Y$ ou vice-versa. Essa coexistência entre duas espécies quimicamente diferentes e o mecanismo envolvido na interconversão é a chave para 0 armazenamento de informações. 


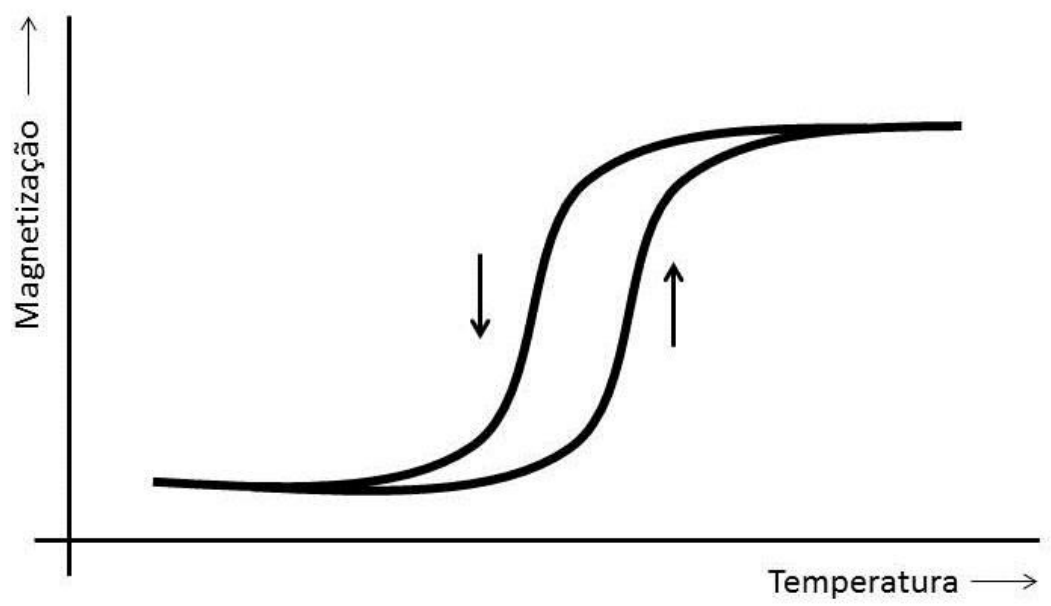

Figura 2. Exemplo genérico de uma histerese

As propriedades atualmente estudadas nos complexos metálicos que podem levar ao desenvolvimento de materiais moleculares eletronicamente lábeis são spin crossover, valência mista e tautomeria de valência. ${ }^{10} \mathrm{~A}$ primeira propriedade refere-se à mudança de multiplicidade do spin de uma transição do íon metálico em função da temperatura, pressão, luz ou composição, como pode ser visto na Figura 3, que representa a transição entre os dois estados de spin em um campo octaédrico. $^{12}$ Teoricamente, é possível observar esse fenômeno em complexos com configuração $d^{4}-d^{7}$, sendo mais comum em complexos de ferro(II) e ferro(III) $\left(d^{5}\right.$ e $d^{6}$, respectivamente), e, em menor proporção, em complexos de cobalto(II) $\left(d^{7}\right) \cdot{ }^{13}$ Os ligantes mais empregados nos compostos que exibem o fenômeno de spin crossover possuem átomos de nitrogênio doadores, como a 1,10-fenantrolina, o isotiocianato e as piridinas substituídas. ${ }^{14}$ Além disso, complexos de ferro(II) com ligantes derivados da 2,6-bis(pirazol-3-il)piridina foram extensivamente estudados por apresentarem spin crossover. ${ }^{15}$

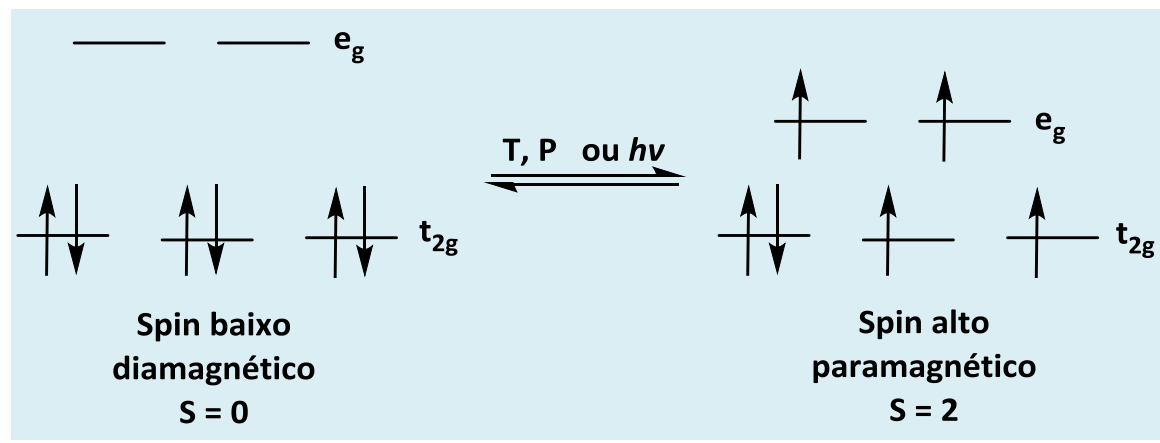

Figura 3. Spin Crossover de dois estados de spin para uma geometria octaédrica com configuração $d^{6}$

Valência mista é uma propriedade que pode ser observada em compostos que possuem pelo menos dois centros redox ativos com diferentes estados de oxidação, conectados por um ligante que atua como ponte permitindo a transferência de elétrons de um íon metálico para outro. A Figura 4 mostra um exemplo de uma molécula que apresenta valência mista. ${ }^{16}$ 
de Morais, A. C. P.

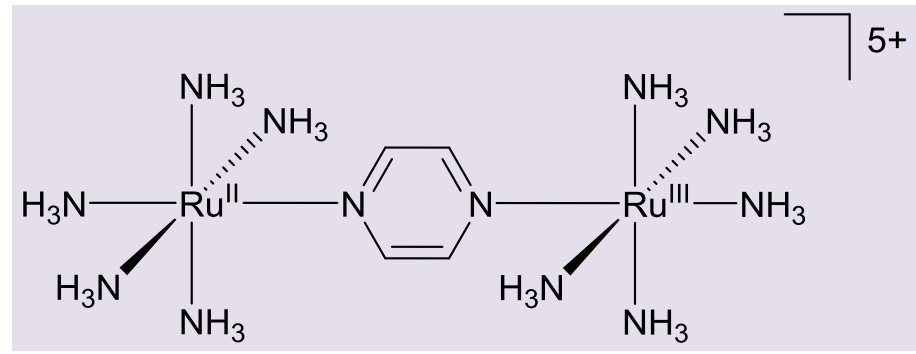

Figura 4. Exemplo de um íon complexo que apresenta valência mista. ${ }^{14}$

A tautomeria de valência, que será abordada neste artigo, é uma propriedade caracterizada pela transferência intramolecular reversível de um elétron, estimulada por meio de uma perturbação externa, envolvendo dois centros redox ativos. $^{10}$ Durante este processo são observados dois isômeros redox, também chamados de tautômeros, ${ }^{17}$ como pode ser visto na Figura 5. Diferentes fatores influenciam a observação de TV, como o ligante eletroativo, os ligantes nitrogenados auxiliares, o estímulo externo e a natureza do íon metálico. Diversas pesquisas estão sendo realizadas com o intuito de desenvolver um sistema ligante-metal que apresente transições características de TV, que possa atuar como um dispositivo molecular.

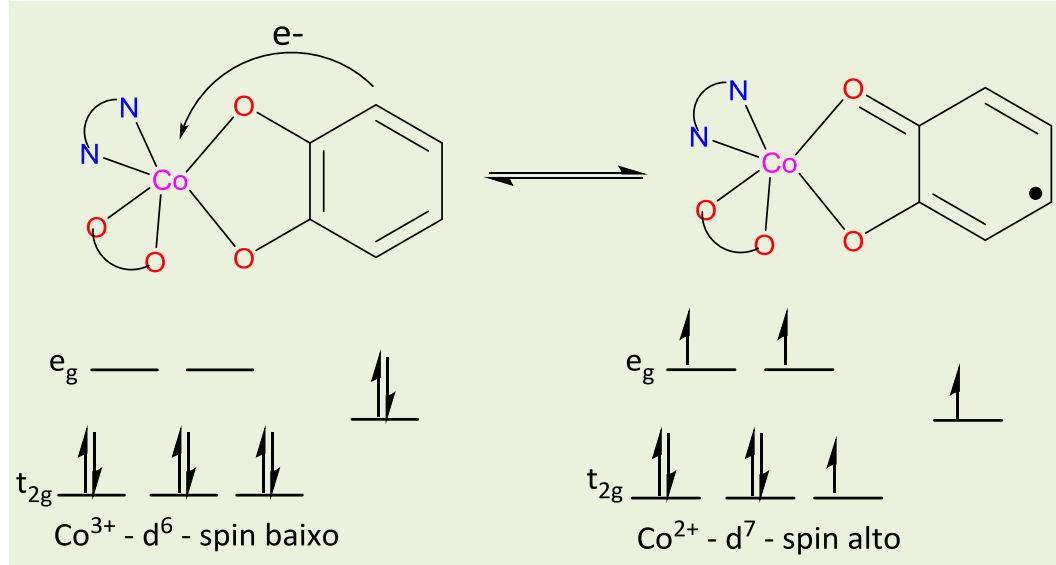

Figura 5. Exemplo genérico de tautomeria de valência

\section{Tautomeria de valência em complexos de cobalto}

O primeiro complexo a apresentar TV foi descrito na literatura em $1980,{ }^{18}$ em que o complexo $\quad\left[\mathrm{Co}\left(2,2^{\prime}\right.\right.$-bpy)(3,5-DTBSQ)(3,5DTBCat)] foi estudado (Figura 6), sendo 2,2'bpy referente ao 2,2'bipiridino, e 3,5-DTBSQ e 3,5-DTBCat significam, respectivamente, as formas semiquinona e catecolato do ligante 3,5-di-terc-butilbenzoquinona. 


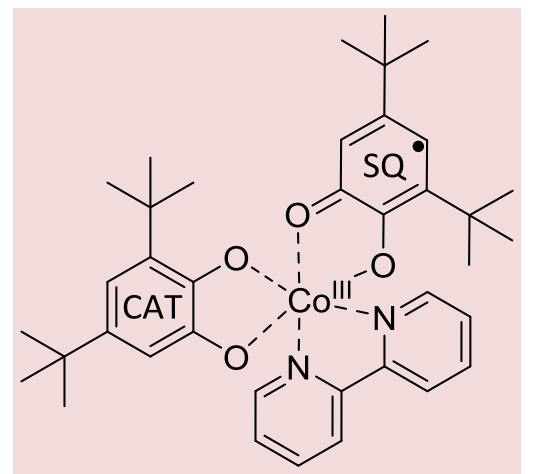

Figura 6. Estrutura do primeiro complexo a apresentar TV descrita na literatura

Desde então, diversas pesquisas foram realizadas usando o 3,5-di-tercbutilbenzoquinona (3,5-DTBQ) (1), ${ }^{19-22}$ o 3,6di-terc-butilbenzoquinona (3,6-DTBQ) (2), ${ }^{23,24}$ e o (2-hidroxi-3,5-di-terc-butilfenil)imino-4,6di-terc-butilciclohexano-3,5-dienona (3), ${ }^{25,26}$ como ligantes redox ativos, representados na Figura 7. Complexos de cobalto com algum destes ligantes tetrassubstituídos com cloro ou bromo (4) também podem ser encontrados na literatura. ${ }^{27,28}$ Estes ligantes são muito utilizados, por serem eletroativos e podem, portanto, existir em três diferentes estados de oxidação, sendo eles: quinona, semiquinona e catecolato. Uma molécula de quinona pode ser reduzida por um elétron, gerando a semiquinona ( $\left.\mathrm{SQ}^{-}\right)$, e também pode ser reduzida por dois elétrons, gerando a forma catecolato $\left(\mathrm{Cat}^{2-}\right.$ ), como pode ser observado na Figura 8.<smiles>CC(C)(C)c1cc(O)c(O)c(C(C)(C)C)c1</smiles>

(1)<smiles>CC(C)(C)C1=CC(=Nc2cc(C(C)(C)C)cc(C(C)(C)C)c2O)C(=O)C(C(C)(C)C)=C1</smiles>

(3)<smiles>CC(C)(C)c1ccc(C(C)(C)C)c(O)c1O</smiles>

(2)<smiles>[X]c1c([X])c(O)c([X])c(O)c1[X]</smiles>

(4) $\mathrm{X}=\mathrm{Cl}$ ou $\mathrm{Br}$

Figura 7. Ligantes redox ativos mais utilizados em complexos que apresentam TV

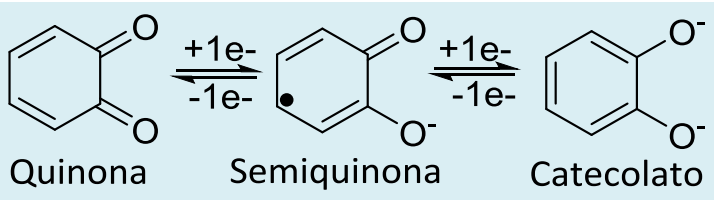

Figura 8. Processo redox da quinona 
Além disso, é necessário que o composto a ser estudado possua simetria adequada e energia próxima entre os orbitais do metal e ligante para que possibilitem a ocorrência de $\mathrm{TV}$, e o cobalto é o íon metálico que possui melhor compatibilidade com estes ligantes. ${ }^{29}$

Em complexos mononucleares 1:2 cobalto:ligante eletroativo, um dos ligantes não participa do fenômeno. ${ }^{17}$ Os complexos [Co'ldpa)(3,5-DTBSQ)(3,5-DTBCat)] e [Co'"(phen)(3,5-DTBSQ)(3,5-DTBCat)] $\cdot \mathrm{C}_{6} \mathrm{H}_{5} \mathrm{Cl}$, estudados por Sato e colaboradores, ${ }^{21,30}$ são exemplos deste tipo de sistema 1:2. ${ }^{18}$ Hendrickson e Adams prepararam uma série de complexos 1:2 com diversos ligantes bidentados $\mathrm{N}-\mathrm{N}$ e todos eles apresentaram TV. $^{19}$ O ligante auxiliar nitrogenado possui uma considerável influência no equilíbrio entre os isômeros redox, sendo capaz de estabilizar o tautômero de cobalto(II) gerado em razão da sua capacidade $\sigma$-doadora. ${ }^{19} \mathrm{O}$ complexo [Co(tmeda)(3,6-DTBSQ)(3,6DTBCat)], estudado por Pierpont e colaboradores, apresenta uma temperatura de transição $200 \mathrm{~K}$ maior do que o complexo com o ligante tmpda, em que tmeda e tmpda significam, $\quad N, N, N^{\prime}, N^{\prime}-$ tetrametiletilenodiamina e $N, N, N^{\prime}, N^{\prime}$ tetrametilpropilenodiamina,

respectivamente. A adição do grupo metileno no anel quelante do ligante nitrogenado tem considerável efeito na transferência eletrônica requerida no fenômeno de TV. ${ }^{31}$ Também há compostos com o 3,6-DTBQ, 32,33 porém, o 3,5-DTBQ é mais comumente usado nos complexos 1:2. A Figura 9 mostra alguns dos ligantes $N-N$ utilizados neste sistema.

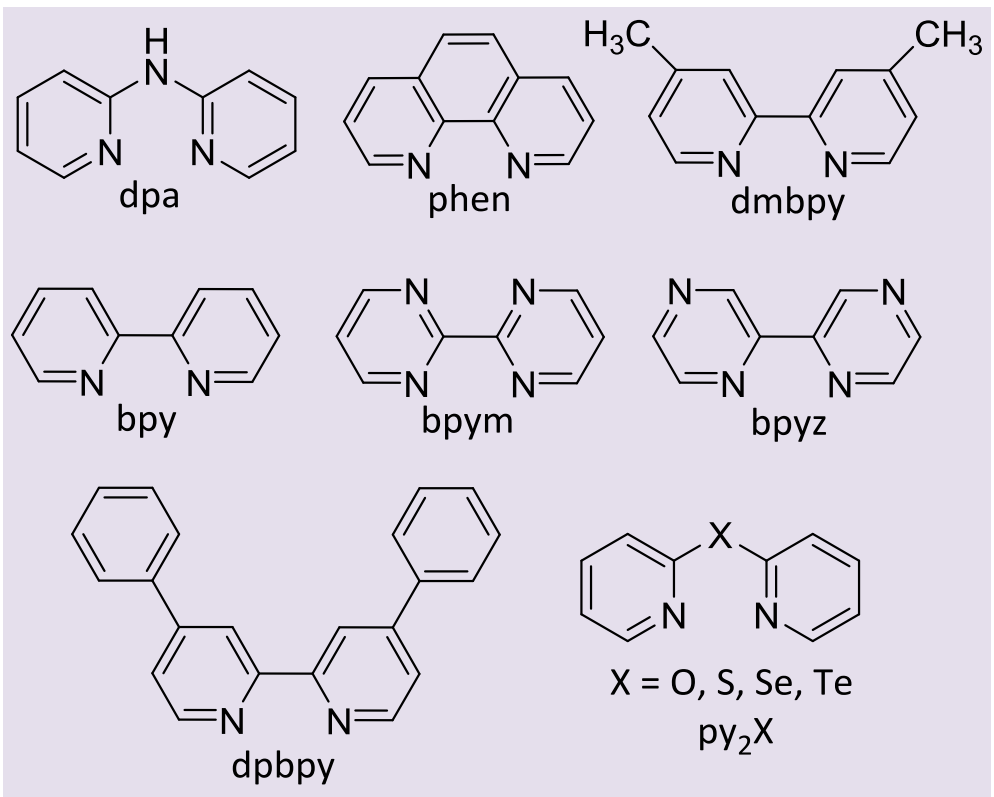

Figura 9. Ligantes nitrogenados auxiliares bidentados utilizados em complexos 1:2 $2^{18,19,21,30,32}$

No caso dos compostos $1: 1$, os mais investigados constituem uma série de complexos de cobalto com ligantes derivados do $\mathrm{Me}_{n}$ TPA ( $\left.\mathrm{n}=0,1,2,3\right)$, em que TPA representa tris-(2-piridilmetil)amina, ${ }^{34-37}$ cuja estrutura do complexo pode ser vista na Figura 10. Transições intramoleculares fotoinduzidas foram observadas para [Co(Me ${ }_{n}$ TPA $)(3,5-$ DTBCat $\left.)\right] \mathrm{PF}_{6}(\mathrm{n}=0,1,2)$ em uma temperatura de $9 \mathrm{~K}$. Além disso, o complexo [Co(Me ${ }_{2}$ TPA)(DTBCat) ${ }^{+}$exibe um começo de TV termoinduzido em aproximadamente $280 \mathrm{~K} .^{38}$ 


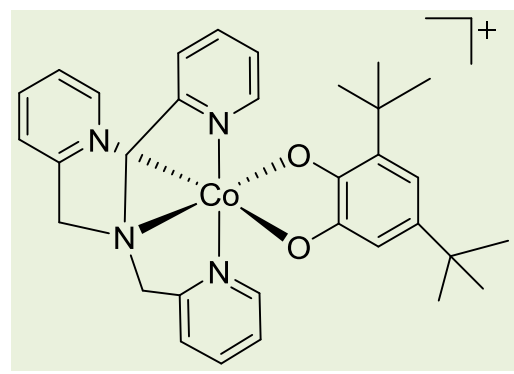

Figura 10. Estrutura do complexo com ligante TPA

\section{Estímulos externos que induzem a tautomeria de valência}

Para que ocorra a transferência eletrônica entre os dois tautômeros, diversos estímulos externos podem ser usados, como temperatura, luz ou pressão. ${ }^{37-39} \mathrm{Um}$ exemplo notável de TV induzido por temperatura é o primeiro complexo a apresentar este fenômeno, ${ }^{18}$ mostrado anteriormente na Figura 6. Em solução de tolueno, em temperaturas acima de $0^{\circ} \mathrm{C}$, o composto apresenta-se na forma de $\mathrm{Co}(\mathrm{III})$, e tal fato pode ser visto no espectro eletrônico, em que uma banda em $610 \mathrm{~nm}$ característica do isômero [Co' (2,2'-bpy)(3,5-DTBSQ)(3,5DTBCat)] pode ser observada. Com o aumento da temperatura, a intensidade da banda em $610 \mathrm{~nm}$ diminui, e uma banda em $770 \mathrm{~nm}$, característica do isômero [Co" $\left(2,2^{\prime}\right.$ bpy)(3,5-DTBCat $)_{2}$, aumenta de intensidade, indicando a ocorrência de interconversão entre os dois isômeros. Medidas de susceptibilidade magnética, RPE (Ressonância Paramagética Eletrônica) e RMN (Ressonância Magnética Nuclear) ajudam a comprovar a existência do equilíbrio entre as espécies $\mathrm{Co}^{3+} / \mathrm{CO}^{2+}$, mostrando que o complexo estudado apresenta TV induzido por temperatura. ${ }^{18}$ Após esta constatação, vários compostos que apresentaram TV foram descritos na literatura, como o primerio complexo a apresentar TV no estado sólido, em que o composto [Co" $(3,5-$ DTBSQ) ${ }_{2}$ (phen)] $\mathrm{CH}_{3} \mathrm{C}_{6} \mathrm{H}_{5}$ (phen significa 1,10 fenantrolina) estudado por Hendrickson e colaboradores em 1993 apresentou uma transição entre os isômeros de $\mathrm{Co}^{2+} \mathrm{e} \mathrm{Co}^{3+}$ no intervalo de temperatura de 220 a $260 \mathrm{~K}^{19}$

Além da temperatura, é possível induzir a transferência de carga através de irradiação de luz em uma dada temperatura. Compostos que apresentam spin crossover podem exibir efeito LIESST (light-induced excited state spin trapping), ${ }^{16,40}$ entretanto, este efeito ainda não foi reportado em complexos com TV, embora casos fotoinduzidos sejam amplamente observados. ${ }^{21,30} \mathrm{O}$ primeiro tautômero de valência fotoinduzido foi estudado por Adams e Hendrickon em $1995,{ }^{41}$ e, desde então, diversos complexos com esta propriedade foram investigados extensivamente. $^{42-45} \mathrm{Um}$ ótimo exemplo deste sistema foi reportado por Cui e Sato, em que o complexo $\left[\mathrm{Co}^{\mathrm{II}-\mathrm{Hs}}(3,5-\right.$ DTBSQ $)_{2}$ (phen)] $\left(\mathrm{C}_{6} \mathrm{H}_{5} \mathrm{Cl}\right)$ predominantemente observado por volta de $300 \mathrm{~K}$, enquanto que o complexo [Co ${ }^{\mathrm{III} L \mathrm{LS}}(3,5-$ DTBCat)(3,5-DTBSQ)(phen)] $\left(\mathrm{C}_{6} \mathrm{H}_{5} \mathrm{Cl}\right)$ é predominantemente observado perto de 11 K. Quando o complexo $\left[\mathrm{Co}^{\text {III-LS }}(3,5-\right.$ DTBCat)(3,5-DTBSQ)(phen)]. $\left(\mathrm{C}_{6} \mathrm{H}_{5} \mathrm{Cl}\right)$ foi irradiado utilizando um laser $\mathrm{Nd}-\mathrm{YAD}$ com um comprimento de onda de $532 \mathrm{~nm}$ em uma temperatura de $5 \mathrm{~K}$, o valor de $\mu_{\text {eff }}$ (momento magnético efetivo) aumentou de 1,7 para 2,7 $\mu_{B}$ em 1 minuto. Isso significa que o estado $\left[\mathrm{Co}^{11-H S}(3,5-\mathrm{DTBSO})_{2}\right.$ (phen)] $\cdot\left(\mathrm{C}_{6} \mathrm{H}_{5} \mathrm{Cl}\right)$ foi gerado pela luz e, em razão da sua rápida resposta à irradiação de luz, este complexo pode ser um candidato a ser utilizado como dispositivo molecular óptico. $O$ fotoprocesso pode ser expresso como [Co"'(3,5-DTBCat)(3,5DTBSQ)(phen) $] \cdot\left(\mathrm{C}_{6} \mathrm{H}_{5} \mathrm{Cl}\right) \rightarrow[\mathrm{Co}$ " $(3,5-$ TBSQ $)_{2}$ (phen)] $\cdot\left(\mathrm{C}_{6} \mathrm{H}_{5} \mathrm{Cl}\right){ }^{30} \mathrm{O}$ complexo análogo [Co ${ }^{\text {III-LS }}(3,5-D T B C a t)(3,5-D T B S Q)($ phen)] 
também apresentou TV fotoinduzida, em que o tempo de vida do isômero $\mathrm{Co}^{\mathrm{II} H \mathrm{H}}$ foi de aproximadamente 600 ns em 190,4 K. ${ }^{8}$ Por outro lado, alguns compostos que exibiram TV fotoinduzida possuem um tempo de vida relativamente longo, ${ }^{21,46}$ como é o caso do $\left[\mathrm{Co}^{11-H s}(3,5-\mathrm{DTBSO})_{2}\right.$ (tmeda) $]$, onde em $5 \mathrm{~K}$, o tempo de vida foi de $175 \mathrm{~min}^{45}$

Complexos de cobalto com TV induzida por temperatura também apresentam, muitas vezes, transição fotoinduzida, ${ }^{43}$ como é o caso do equilíbrio mostrado na Figura 11, que pode ser monitorado por meio de susceptibilidade magnética após a irradiação de uma luz verde no complexo de cobalto(III), a uma temperatura de $5 \mathrm{~K}$. Estudos de espectroscopia na região do infravermelho com dependência de temperatura também fornecem um suporte adicional para confirmar a ocorrência de TV, além de medidas magnéticas. Este sistema pode ser um bom candidato para a aplicação em eletrônica molecular em virtude das diversas formas de se obter transições eletrônicas. ${ }^{23} \mathrm{O}$ composto [Co" $(\mathrm{dpa})(3,5-\mathrm{DTBSQ})_{2}$ ] estudado por Sato e colaboradores também apresentou TV induzida por temperatura e por luz. A transição entre os isômeros foi observada em uma temperatura de $380 \mathrm{~K}$, e uma irradiação de luz a $5 \mathrm{~K}$ monitorada por medidas magnéticas, também induziu a transferência eletrônica entre os tautômeros. ${ }^{21}$

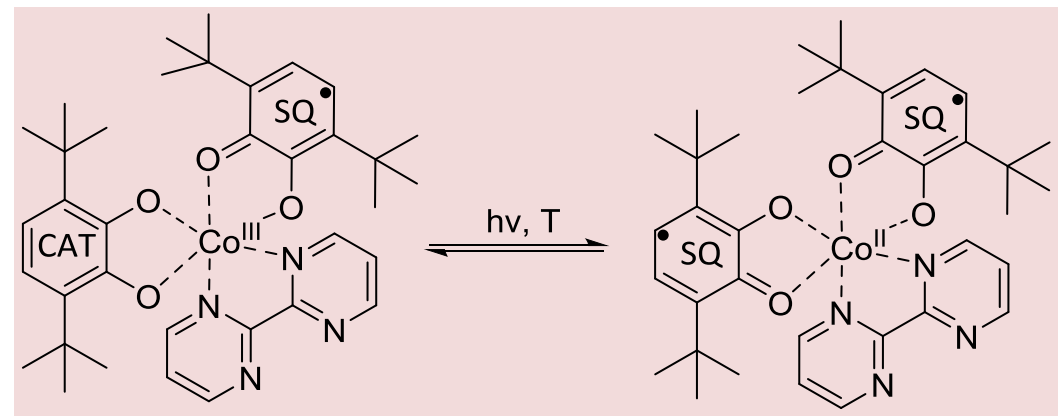

Figura 11. Equilíbrio induzido por temperatura e por luz estudado por Sato e colaboradores ${ }^{23}$

Outra forma de induzir transições eletrônicas intramoleculares é por meio de pressão, ${ }^{39,47}$ embora em menor extensão do que os casos térmicos ou fotoinduzidos reportados, principalmente por dificuldades experimentais. Na década de 1990, foram descritos os primeiros compostos que apresentam tautomeria de valência induzida por pressão reversível. Os complexos estudados foram [Co"(3,5-DTBSQ) 2 (phen)] e [Co"(3,5-DTBSQ $)_{2}$ (phen)]. $\mathrm{C}_{6} \mathrm{H}_{5} \mathrm{CH}_{3}$, e a TV pôde ser detectada por EXAFS (Extended $X$ Ray Absorption Fine Structure) e XANES ( $X$ ray Absorption Near Edge Structure). ${ }^{48}$

Entretanto, para que os compostos de cobalto possam ser usados como dispositivos moleculares, como os de armazenamento de informações, é desejável que os complexos apresentem histerese em temperatura ambiente, $^{43}$ possibilitando que o composto possua dois estados diferentes. Assim, o composto teria um estado "ligado" e outro "desligado", como nos dispositivos de memória e armazenamento. O complexo [Co'l'(3,6-DTBCat)(3,6-DTBSO) $\left.\left(\mathrm{py}_{2} \mathrm{O}\right)\right] \quad\left(\mathrm{py}_{2} \mathrm{O}\right.$ significa 2,2'-bis(piridina)éter) estudado por Pierpont apresenta, em altas temperaturas, a transição $\mathrm{Co}^{3+} \rightarrow \mathrm{Co}^{2+}$ com uma $\mathrm{T}_{\mathrm{c}} \uparrow$ de 330 $\mathrm{K}$, e a transição reversa $\mathrm{Co}^{2+} \rightarrow \mathrm{Co}^{3+}$ é observada em uma $T_{c} \downarrow$ de $100 \mathrm{~K}$, sendo possível observar uma larga histerese de 230 $K$, em que $T_{c}$ significa temperatura crítica. $A$ histerese térmica associada a uma transferência eletrônica é observada em razão das interações intermoleculares que ocorrem dentro da rede cristalina. ${ }^{32}$ Mais especificamente, as ligações de hidrogênio podem estabilizar o tautômero de cobalto(II) gerado por meio de um mecanismo que facilita a redução da barreira de energia do 
alongamento das distâncias de ligação do cobalto(II) com os átomos de nitrogênio ou oxigênio. $^{49}$

A existência da ampla histerese é fundamental para a possível aplicação em dispositivos de armazenamento de informação. Outros compostos descritos na literatura apresentam esta característica, ${ }^{50-52}$ mas ainda é preciso buscar novos compostos que possuam uma histerese larga o suficiente para uma aplicação prática. ${ }^{42}$

\section{Outros metais na tautomeria de valência}

Apesar dos complexos de cobalto serem os mais amplamente estudados em sistemas com TV, transições intramoleculares também podem ser observadas em outros metais. Compostos de manganês são ótimos candidatos a TV. ${ }^{53-55}$ Diferentemente dos complexos de cobalto, não apresentam mudança de spin baixo para spin alto após a transferência de elétron, ou seja, permanecem no estado de spin alto. Além disso, o manganês possui três estados de oxidação: $\mathrm{Mn}^{4+}, \mathrm{Mn}^{3+}$ e $\mathrm{Mn}^{2+}$ acessíveis. ${ }^{56} \mathrm{~A}$ ocorrência de tautomeria de valência induzida por temperatura, monitorada por susceptibilidade magnética, foi observada no complexo [Mn(cth)(3,5-DTBCat)]X (X $=\mathrm{BPh}_{4}$ ou $\mathrm{ClO}_{4} ;$ cth $=1-5,7,7,12,14,14$-hexametil1,4,8,11-tetraazaciclotetradecano). Nesse composto, as propriedades espectroscópicas são dependentes da temperatura, ou seja, os complexos mudam de cor ao serem submetidos ao aquecimento ou ao resfriamento e, em temperatura ambiente, voltam à cor original em poucos minutos. ${ }^{57}$

Embora os complexos de ferro sejam muito estudados por apresentarem spin crossover, o primeiro composto de ferro a exibir TV só foi reportado em 2004, e sua estrutura pode ser vista na Figura 12. O composto estudado por Shaikh apresentou uma transferência eletrônica intramolecular termoinduzida tanto no estado sólido como em solução do tipo $\mathrm{Fe}^{3+} \rightarrow \mathrm{Fe}^{2+}$, similar à dos complexos de cobalto e manganês, observada no espectro eletrônico e em medidas magnéticas. ${ }^{58}$

Além do cobalto, manganês e ferro, TV também foi observada em complexos de $\mathrm{Ni}^{59,60} \mathrm{Cu}^{61}$ e Ru. ${ }^{62}$

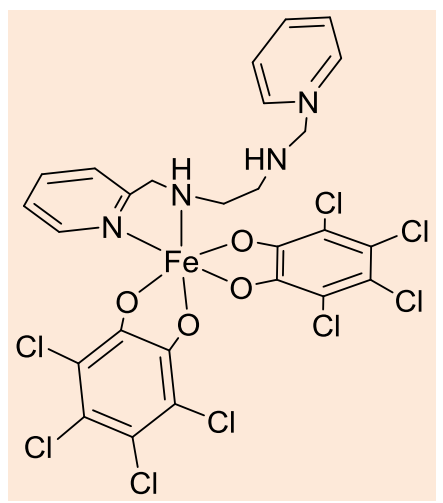

Figura 12. Estrutura do primeiro complexo de ferro a apresentar $\mathrm{TV}^{58}$

\section{Considerações finais}

Desde o primeiro complexo a exibir TV em 1980, diversos pesquisadores vêm reportando sistemas com tautomeria de valência induzida por temperatura, luz e em menor proporção com pressão, em alguns casos com uma histerese pronunciada. Um conjunto de fatores pode influenciar a faixa de temperatura na ocorrência da 
interconversão entre os dois isômeros, como o íon metálico, o ligante nitrogenado auxiliar, o ligante redox ativo, a temperatura crítica, dentre outros. Além disso, a ocorrência de TV no estado sólido é limitada e alguns complexos relatados na literatura apresentam uma temperatura de transição baixa, principalmente aqueles fotoinduzidos, - que dificulta uma eventual aplicação prática. Desse modo, a busca por um composto que apresente TV e possa as características adequadas para ser aplicado como um dispositivo molecular continua sendo um desafio.

\section{Referências Bibliográficas}

${ }^{1}$ Toma, H. E.; O mundo nanométrico: a dimensão de novo século. Oficina de textos, São Paulo, 2004.

${ }^{2}$ Rezende, S. M. A aventura da física da matéria condensada. Ciência Hoje 2005, 37, 26. [Link]

${ }^{3}$ Moore, G. E. Cramming more components onto integrated circuits. Electronics 1998, 86, 82. [CrossRef]

${ }^{4}$ Schaller, R. R. Moore's Law: Past, Present, and Future. IEEE Spectrum 1997, 34, 52. [CrossRef]

${ }^{5}$ Kanellos, M. Intel scientists find wall for Moore's Law. CNET. Disponível em: $<$ http://news.cnet.com/Intel-scientists-findwall-for-Moores-Law/2100-1008 3-

5112061.html>. Acesso em: 30 setembro 2014.

${ }^{6}$ Ratner, M. A.; Aviram, A. Molecular rectifiers. Chemical Physics Letters 1974, 29, 277. [CrossRef]

${ }^{7}$ Meyer, T. J.; Hartshrn, C. M.; Demadis, K. D. The Localized-to-Delocalized Transition in Mixed-Valence Chemistry. Chemical Reviews 2001, 101, 2655. [CrossRef] [PubMed]

${ }^{8}$ Adams, D. M.; Hendrickson, D. N. Pulsed Laser Photolysis and Thermodynamics Studies of Intramolecular Electron Transfer in Valence Tautomeric Cobalt o-Quinone Complexes. Journal of the American Chemical Society 1996, 118, 11515. [CrossRef]
${ }^{9}$ Evangelio, E.; Rodriguez-Blanco, C.; Coppel, Y.; Hendrickson, D. N.; Sutter, J. P.; Campo, J.; Ruiz-Molina, D. Solvent effects on valence tautomerism: A comparison between the interconversion in solution and solid state. Solid State Sciences 2009, 11, 793. [CrossRef]

${ }^{10}$ Schultz, D. A. Valence Tautomerism in Dioxolene Complexes of Cobalt. Magnestism: Molecules to Materials II; Miller, J. S. Drillon, M. eds.; Wiley: New York, 2002.

${ }^{11}$ Bin-Salamon, S.; Brewer, S.; Franzen, S.; Feldheim, D. L.; Lappi, S.; Schultz, D. A. Supramolecular control of valencetautomeric equilibrium on nanometer-scale gold clusters. Journal of the American Chemical Society Communications 2005, 127, 5328. [CrossRef] [PubMed]

${ }^{12}$ Larionov, S. V. Spin transition in iron(III) and iron(II) complexes. Russian Journal of Coordination Chemistry 2008, 34, 237. [CrossRef]

${ }^{13}$ Kahn, O.; Martinez, C. J.; Spin-transition polymers: from molecular materials toward memory devices. Science 1998, 279, 44. [CrossRef]

${ }^{14}$ Gütlich, P.; Garcia, Y.; Goodwin, H. A. Spin crossover phenomena in $\mathrm{Fe}(\mathrm{II})$ complexes. Chemical Society Reviews 2000, 29, 419. [CrossRef]

${ }^{15}$ Craig, G. A.; Roubeau, O.; Aromí, G. Spin state switching in 2,6-bis(pyrazol-3yl)pyridine (3-bpp) based $\mathrm{Fe}(\mathrm{II})$ complexes. Coordination Chemistry Reviews 2014, 269, 13. [CrossRef]

${ }^{16}$ Creutz, C.; Taube, H. A. Direct approach to measuring the Franck-Condon barrier to electron transfer between metal ions. Journal of the American Chemical Society 1969, 91 (14), 3988. [CrossRef]

${ }^{17}$ Tezgerevska, T.; Alley, K. G.; Boskovic, C. Valence tautomerism in metal complexes: Stimulated and reversible intramolecular electron transfer between metal centers and organic ligands. Coordination Chemistry Reviews 2014, 268, 23. [CrossRef]

${ }^{18}$ Pierpont, C. G.; Buchanan, R. M. Tautomeric catecholate-semiquinone interconversion via metal-ligand electron transfer. Structural, spectral, and magnetic 
properties of (3,5-di-tert-butylcatecholato)(3,5-di-tert-

butylsemiquinone)(bipyridyl)cobalt(III), a complex containing mixed-valence organic ligands. Journal of the American Chemical Society, 1980, 102, 4951. [CrossRef]

${ }^{19}$ Adams, D. M.; Dei, A.; Rheingold, A. L.; Hendrickson, D. N. Bistability in the [Co"(semiquinonate) 2 ]

[Co"'(catecholate)(semiquinonate)] valencetautomeric conversion. Journal of the American Chemical Society 1993, 115, 8221. [CrossRef]

${ }^{20}$ Pierpont, C. G.; Jung, O.-S. Thermodynamic Parameters for Cobalt-Quinone Electron Transfer and Spin Transition Steps of Co'"(bpy)(3,5-DBSQ)(3,5-DBCat)/Co"(bpy)(3,5DBSQ) 2 Valence Tautomeric Equilibrium. Inorganic Chemistry 1995, 34, 4281. [CrossRef]

${ }^{21}$ Cui, A.; Takahashi, K.; Fujishima, A.; Sato, O. Novel Co complex with high transformation temperature of valence tautomerism. Journal of Photochemistry and Photobiology A: Chemistry 2004, 161, 243. [CrossRef]

${ }^{22}$ Kadohashi, Y.; Maruta, G.; Taketa, S. Spin and charge fluctuation occurring in valence tautomerism and cooperative effects in the solid-state. Polyhedron 2007, 26, 2342. [CrossRef]

${ }^{23}$ Dai, J.; Kanegawa, S.; Li, Z.; Kang, S.; Sato, O. A switchable complex ligand exhibiting photoinduced valence tautomerism. European Journal Inorganic Chemistry 2013, 24, 4150. [CrossRef]

${ }^{24}$ Attla, A. S.; Pierpont, C. G. New semiquinone/catecholate complexes that exhibit valence tautomerism. synthesis and characterization of $\mathrm{Mn}^{\text {III }}$ (thf) $)_{2}$ (3,6-DBSQ)(3,6DBCat) and observations on the $\operatorname{Mn}^{\mathrm{IV}}(3,6$ DBSQ $)_{2}(3,6-\mathrm{DBCat}) / \mathrm{Mn}^{\prime \prime \prime}(3,6-\mathrm{DBSQ})_{3}$.

Equilibrium in the solid state. Inorganic Chemistry 1998, 37, 3051. [CrossRef]

${ }^{25}$ Cador, O.; Chabre, F.; Dei, A.; Sangregorio, C.; Slageren, J. V.; Vaz, M. G. F. Temperatureinduced solid-state valence tautomeric interconversion in two cobalt-schiff base diquinone complexes. Inorganic Chemistry 2003, 42, 6432. [CrossRef] [PubMed]

${ }^{26}$ Beni, A.; Carbonera, C.; Dei, A.; Létard, J-F.; Righini, R.; Sangregorio, C.; Sorace, L. Optically induced valence tautomeric interconversion in cobalt dioxolene complexes. Journal of the Brazilian Chemical Society 2006, 17, 1522. [CrossRef]

${ }^{27}$ Dei, A.; Feis, A.; Poneti, G.; Sorace, L. Thermodynamics of valence tautomeric interconversion in a tetrachlorodioxolene:cobalt 1:1 adduct. Inorganica Chimica Acta 2008, 361, 3842. [CrossRef]

${ }^{28}$ Panja, A. A series of tetrabromocatecholate chelated cobalt(III) complexes with various $\mathrm{N}$ donor ancillary ligands: syntheses, crystal structures, co-crystallization, thermally induced valence tautomerism and electrochemical studies. RSC Advances 2013, 3, 4954. [CrossRef]

${ }^{29}$ Evangelio, E.; Ruiz-Molina, D. Valence tautomerism: More actors than just electroactive ligands and metal ions. Comptes Rendus Chimie 2008, 11, 1137. [CrossRef]

${ }^{30}$ Cui, A.; Takahashi, K.; Fujishima, A.; Sato, 0 . Mechanism and relaxation kinetics of photo-induced valence tautomerism of [Co(phen)(3,5-DBSQ) $\left.{ }_{2}\right] \cdot \mathrm{C}_{6} \mathrm{H}_{5} \mathrm{Cl}$. Journal of Photochemistry and Photobiology A: Chemistry 2004, 167, 69. [CrossRef]

${ }^{31}$ Jung, O.-S.; Jo, D. H.; Lee, Y.-A; Sohn, Y. S.; Pierpont, C. G. Ancillary ligand dependent shifts in charge distribution for cobaltquinone complexes. Angewandte Chemie International Edition 1996, 35, 1694. [CrossRef]

32 Jung, O.-S.; Jo, D. H.; Lee, Y.-A.; Conklin, B. J.; Pierpont, C. G. Bistability and molecular switching for semiquinone and catechol complexes of cobalt. studies on redox isomerism for the bis(pyridine) ether series $\mathrm{Co}\left(\mathrm{py}_{2} \mathrm{X}\right)(3,6-\mathrm{DBQ})_{2}, \mathrm{X}=\mathrm{O}, \mathrm{S}, \mathrm{Se}$, and Te. Inorganic Chemistry 1997, 36, 19. [CrossRef]

${ }^{33}$ Pierpont, C. G. Studies on charge distribution and valence tautomerism in transition metal complexes of catecholate and semiquinonate ligands. Coordination Chemistry Reviews 2001, 216-217, 99. [CrossRef] 
${ }^{34}$ Dei, A.; Sorace, L. Cobalt-dioxolene redox isomers: potential spintronic devices. Applied Magnetic Resonance 2010, 38, 139. [CrossRef]

${ }^{35}$ Tourón, P. T.; Mosquera, S. M.; Lima, M.; Lapini, A.; Foggi, P.; Dei, A.; Righini, R. Transient infrared spectroscopy: a new approach to investigate valence tautomerism. Physical Chemistry Chemical Physics 2012, 14, 1038. [CrossRef] [PubMed]

${ }^{36}$ Poneti, G.; Mannini, M., Sorace, L.; Sainctavit, P.; Arrio, M.-A.; Otero, E.; Cezar, J. C.; Dei, A. Soft-X-ray-induced redox isomerism in a cobalt dioxolene complex. Angewandte Chemie International Edition 2010, 49, 1954. [CrossRef] [PubMed]

${ }^{37}$ Beni, A.; Dei, A.; Rizzitano, M.; Sorace, L. Unprecedented optically induced long-lived intramolecular electron transfer in cobaltdioxolene complexes. Chemical Communication 2007, 2160. [CrossRef] [PubMed]

${ }^{38}$ Beni, A.; Dei, A.; Laschi, S.; Rizzitano, M.; Sorace, L. Tuning the charge distribution and photoswitchable properties of cobaltdioxolene complexes by using molecular techniques. Chemistry - A European Journal 2008, 14, 1804. [CrossRef] [PubMed]

${ }^{39}$ Caneschi, A.; Dei, A.; Biani, F. F.; Gütlich, P.; Ksenofontov, V.; Levchenko, G.; Hoefer, A.; Renz, F. Pressure- and temperature-induced valence tautomeric interconversion in a 0 dioxolene adduct of a cobalt tetraazamacrocycle complex. Chemistry - A European Journal 2001, 7, 3926. [CrossRef]

${ }^{40}$ Hayami, S.; Gu, Z.-z.; Shiro, M.; Einaga, Y.; Fujishima, A.; Sato, O. First observation of light-induced excited spin state trapping for an iron(ii) complex. Journal of the American Chemical Society 2000, 122, 7126. [CrossRef]

${ }^{41}$ Adams, D.; Li, B.; Simon, J. D.; Hendrickson, D. N. Photoinduced valence tautomerism in cobalt complexes containing semiquinone anion as ligand: dynamics of the high-spin [Co" $\left.(3,5-\mathrm{dtbsq})_{2}\right]$ to low-spin [Co"'(3,5dtbsq)(3,5-dtbcat)] interconversion. Angewandte Chemie International Edition 1995, 34, 1481. [CrossRef]

${ }^{42}$ Sato, O.; Tao, J.; Zhang, Y.-Z. Control of magnetic properties through external stimuli.
Angewandte Chemie International Edition 2007, 46, 2152. [CrossRef] [PubMed]

${ }^{43}$ Sato, O.; Cui, A.; Matsuda, R.; Tao, J.; Hayami, S. Photo-induced valence tautomerism in Co complexes. Accounts of Chemical Research 2007, 40, 361. [CrossRef] [PubMed]

${ }^{44}$ Beni, A.; Dei, A.; Shultz, D. A.; Sorace, L. Ligand design modulates photoinduced properties of cobalt-dioxolene valence tautomers. Chemical Physics Letters 2006, 428, 400. [CrossRef]

${ }^{45}$ Sato, O.; Hayami, S.; Gu, Z-z.; Takahashi, K.; Nakajima, R.; Seki, K.; Fujishima, A. Photoinduced valence tautomerism in a Co compound. Journal of Photochemistry and Photobiology A: Chemistry 2002, 149, 111. [CrossRef]

${ }^{46}$ Sato, O.; Hayami, S.; Gu, Z-z.; Takahashi, K.; Nakajima, R.; Fujishima, A. Photo-induced reverse valence tautomerism in a metastable Co compound. Chemical Physics Letters 2002, 355, 169. [CrossRef]

${ }^{47}$ Li, B.; Yang, F-L.; Tao, J.; Sato, O.; Huang, RB.; Zheng, L-S. The effects of pressure on valence tautomeric transitions of dinuclear cobalt complexes. Chemical Communications 2008, 6019. [CrossRef] [PubMed]

${ }^{48}$ Roux, C.; Adams, D. M.; Itié, J. P.; Polian, A.; Hendrickson, D. N.; Verdaguer, M. Pressureinduced valence tautomerism in cobalt $o$ quinone complexes: An X-ray absorption study of the low-spin [Co"'(3,5-DTBSQ)(3,5DTBCat)(phen)] to high-spin [Co"(3,5DTBSQ) 2 (phen)] interconversion. Inorganic Chemistry 1996, 35, 2846. [CrossRef]

${ }^{49}$ Schmidt, R. D.; Shultz, D. A.; Martin, J. D. Magnetic bistability in a cobalt bis(dioxolene) complex: long-lived photoinducedvalence tautomerism. Inorganic Chemistry 2010, 49, 3162. [CrossRef] [PubMed]

${ }^{50}$ Tao, J.; Maruyama, H.; Sato, O. Valence tautomeric transitions with thermal hysteresis around room temperature and photoinduced effects observed in a cobalttetraoxolene complex. Journal of the American Chemical Society 2006, 128, 1790. [CrossRef] [PubMed]

${ }^{51}$ Caneschi, A.; Cornia, A.; Dei, A. Valence tautomerism in a cobalt complex of a Schiff 
base diquinone ligand. Inorganic Chemistry 1998, 37, 3419. [CrossRef]

${ }^{52}$ Cador, O.; Dei, A.; Sangregorio, C. Isotopic effects may induce cooperativity in valence tautomeric transition. Chemical Communications 2004, 652. [CrossRef] [PubMed]

${ }^{53}$ Goswami, S.; Panja, A.; Butcher, R. J.; Shaikh, N.; Banerjee, P. Ligand-to-ligand electron transfer and temperature induced valence tautomerism in o-dioxolene chelated manganese complexes. Inorganica Chimica Acta 2001, 370, 311. [CrossRef]

${ }^{54}$ Panja, A. Unusual structural features in tetrabromocatechol-chelated dinuclear manganese(III) complex: Synthesis, electrochemistry and thermally induced valence tautomerism. Inorganic Chemitry Communications 2012, 24, 140. [CrossRef]

${ }^{55}$ Shaikh, N.; Goswami, S.; Panja, A.; Sun, HL.; Pan, F.; Gao, S.; Banerjee, P. Syntheses, crystal structures, and spectroscopic and magnetic properties

$\left[\mathrm{Mn}_{2}{ }^{\prime \prime \prime}\left(\mathrm{H}_{2} \mathrm{~L}^{1}\right)\left(\mathrm{Cl}_{4} \mathrm{Cat}\right) \cdot 2 \mathrm{H}_{2} \mathrm{O}\right]$ of $\left[\mathrm{Mn}_{2}{ }^{\prime \prime \prime}\left(\mathrm{H}_{2} \mathrm{~L}^{2}\right)\left(\mathrm{Cl}_{4} \mathrm{Cat}\right)_{4} \cdot 2 \mathrm{CH}_{3} \mathrm{CN} \cdot 2 \mathrm{H}_{2} \mathrm{O}\right]$ :

temperature-dependent valence tautomerism in solution. Inorganic Chemistry 2005, 44, 9714. [CrossRef] [PubMed]

${ }^{56}$ Lynch, M. W.; Hendrickson, D. N.; Fitzgerald, B. J.; Pierpont, C. G. LigandInduced valence tautomerism in manganesequinone complexes. Journal of the American Chemical Society 1981, 103, 3961. [CrossRef]

${ }^{57}$ Caneschi, A.; Dei, A. Valence Tautomerism in a $O$-Benzoquinone Adduct of a tetraazamacrocycle complex of manganese.
Angewandte Chemie International Edition 1998, 37, 3005. [CrossRef]

${ }^{58}$ Shaikh, N.; Goswami, S.; Panja, A.; Wang, XY.; Gao, S.; Butcher, R. J.; Banerjee, P. New route to the mixed valence semiquinonecatecholate based mononuclear $\mathrm{Fe}^{\mathrm{III}}$ and catecholate based dinuclear $\mathrm{Mn}^{\text {III }}$ complexes: first experimental evidence of valence tautomerism in an iron complex. Inorganic Chemistry 2004, 43, 5908. [CrossRef] [PubMed]

${ }^{59}$ Ohtsu, H.; Tanaka, K. Chemical control of valence tautomerism of nickel(II) semiquinone and nickel(III) catecholate states. Angewandte Chemie International Edition 2004, 43, 6301. [CrossRef] [PubMed] ${ }^{60}$ Ohtsu, H.; Tanaka, K. Electronic structural changes between nickel(II)-semiquinonato and nickel(III)-catecholato states driven by chemical and physical perturbation. Chemistry - A European Journal 2005, 11, 3420. [CrossRef] [PubMed]

${ }^{61}$ Harmalker, S.; Jones, S. E.; Sawyer, D. T. Electrochemical and spectroscopic studies of 3,5-di-tert-butylcatecholato and 3,5-di-tertbutyl-o-semiquinonato complexes of copper(II). Inorganic Chemistry 1983, 22, 2790. [CrossRef]

${ }^{62}$ Biswas, M. K.; Patra, S. C.; Maity, A. N.; Ke, S.-C.; Adhikary, N. D.; Ghosh, P. Electronic structures of ruthenium and osmium complexes of 9,10- phenanthrenequinone. Inorganic Chemistry 2012, 51, 6687. [CrossRef] [PubMed] 